\title{
Effect of Atmospheric Absorption Bands on the Optimal Design of Multijunction Solar Cells
}

William E. McMahon, Daniel J. Friedman, John F. Geisz

National Renewable Energy Laboratory, Golden, Colorado, 80401, USA

\section{Summary:}

Designing terrestrial multijunction (MJ) cells with $5+$ junctions is challenging, in part because the presence of atmospheric absorption bands creates a design space with numerous local maxima.

Here we introduce a new taxonomical structure which facilitates both numerical convergence and the visualization of the resulting designs.

(2) Deviations from Current-Matching (2J Example)

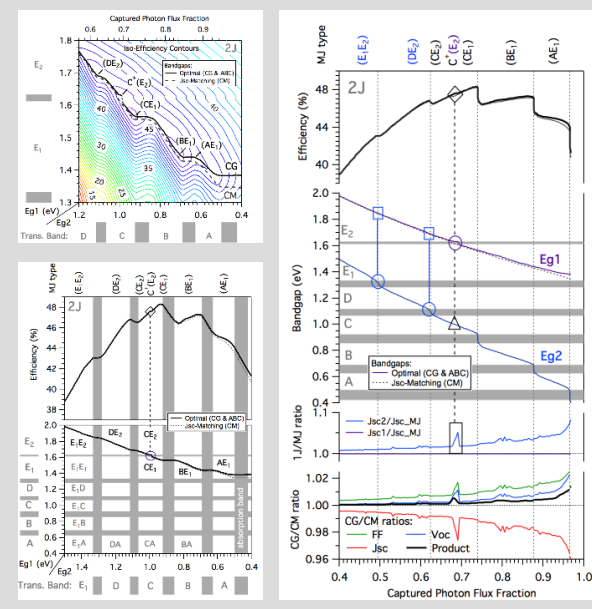

(Upper Left) Iso-efficiency contours. A "Current-Graded" (CG) solution with a slightly raised top-cell bandgap is more efficient than the "Current-Matched" (CM) design. The efficiency maximum for each "MJ type" has been labeled.

(Lower Left) The same information overlain with the transmission band positions, defining the subcell bandgap domains corresponding to each MJ type. All viable MJ types lie near th current-matched line, and can therefore be quickly identified.

(Upper Right) Subcell bandgaps and modeling results plotted against "Captured Photon Flux Fraction" (CF), where CF is the fraction of the photons in the spectrum with energies above the bottom cell bandgap.

For CG solutions, the subcell Jsc increases traveling down through the MJ stack. Although this current mismatch decreases Jsc, both Voc and FF increase such that the product of the three CG/CM ratios is greater than unity.

The anomolous Jsc ratio (bracketed) corresponds to an "Absorption-Band Constrained" (ABC) design. - See Sections 3 and 4.

Note: All calculations shown here are for idealized, optically thick subcells at $300 \mathrm{~K}, 1000$ suns with unity above-bandgap $Q E, n=1$, $R_{\text {series }}=0, R_{\text {shunt }}=\infty, E R E=0.01$, and luminescent coupling $=0$.
(1) A New Taxonomy based on "Transmission Bands"
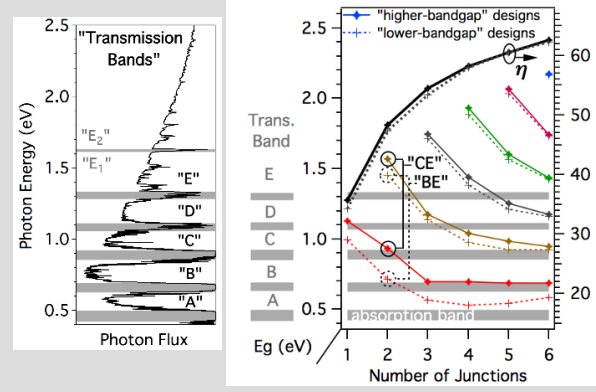
(Right) As the number of junctions increases, numerous nearoptimal designs can be found. Here we propose a taxonomical structure based upon the location of each bandgap with respect to the "Transmission Bands".

Each local efficiency maximum can be assigned a "MJ type" (nomenclature below) which conveys information about subcell current-matching.

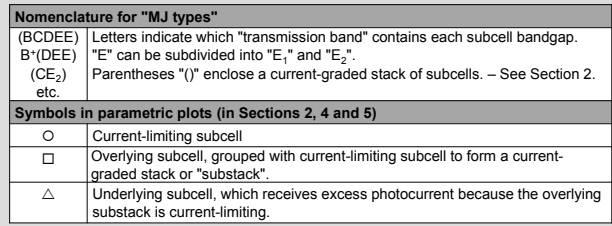

(3) "Absorption-Band Constrained" (ABC) Designs
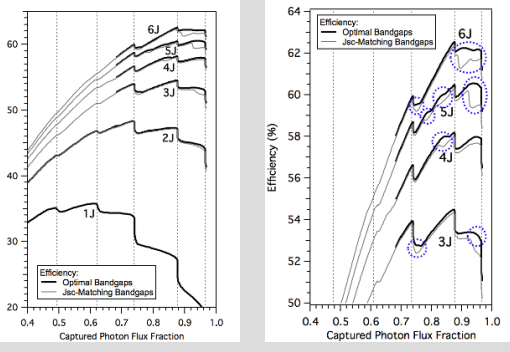

(Left) The MJ cell efficiency drops abruptly wherever the bottom cell bandgap encounters an absorption band (dashed lines), creating numerous local maxima.

(Right) Absorption-band-driven deviations from "currentmatching" (circled) become more significant as the number of junctions increases. These "Absorption-Band Constrained" (ABC) designs occur where an upper-subcell bandgap encounters an atmospheric absorption band.

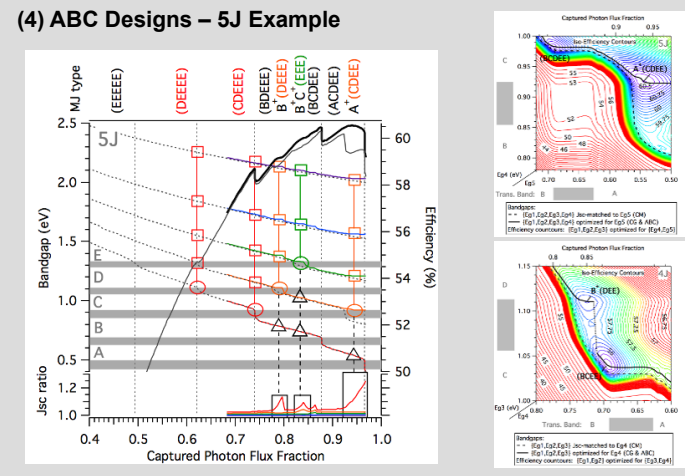

(Left) ABC designs occur where an upper-subcell bandgap (circled) encounters an absorption band. The underlying subcells become over-driven (triangles), with an anomolous Jsc ratios (brackets). The overlying subcells (squares) combine with the current-limiting subcell to form a CG "sub-stack" (parentheses in "MJ type" name, at top). Interestingly, an ABC design, $\mathrm{A}^{+}(\mathrm{CDEE})$, is the most efficient design for this $5 \mathrm{~J}$ example.

(Right) Iso-efficiency plots for subcell bandgaps near two of the $A B C$ designs.
(5) Design Strategy - 6J Example

(Left) Design space for a 6-junction cell. Current-matched (CM) designs are plotted with light grey. CG and ABC designs are shown with black and colored lines.

(Right) Each MJ type supports a single efficiency maximum. This suggests a two-step design strategy:

Step 1. Determine the viable MJ types, based on their proximity to the $\mathrm{CM}$ design.

Step 2. Constrain the subcell bandgap domains for each MJ type such that there is a single efficiency maximum for each MJ type, enabling the use of a fast, gradient-based solver.

This should facilitate numerical convergence, while also providing information about multiple near-optimal designs.

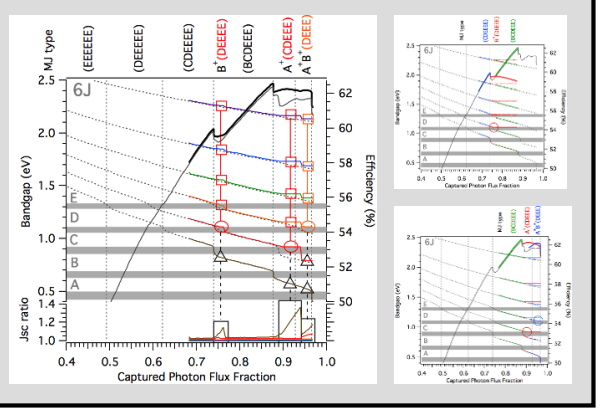

